\title{
Stroke: getting patients to hospital quicker
}

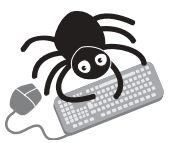

Please visit the Quality and Safety in Health Care website [www. qshc.com] for a link to the full text of this article. reatment for acute stroke must be given as soon as possible after the onset of symptoms if it is to be effective. For many patients there is a significant delay in reaching hospital.

Researchers in Plymouth, UK have looked into three aspects of this delay; the time taken by patients to decide they are seriously ill, the time taken to call for medical help, and the time from calling for medical help to reaching hospital.

They interviewed four groups, each of 40 people, using a standardised, structured interview with open ended questions. The four groups were; patients with stroke or transient ischaemic attack (able to participate and interviewed within 48 hours of admission to hospital), patients at risk of stroke (attending clinics for hypertension, diabetes, or chronic renal failure), members of the general public, and hospital nurses (medical rather than surgical but not working on neurology wards).

Sixteen of the 40 patients $(40 \%)$ realised that they were having a stroke. This proportion was higher $(57 \%)$ among patients who had been told they were at risk than among other patients (35.5\%, difference not significant). The median time from symptom onset to calling for medical help (illness delay) was 30 minutes (range five minutes to nearly six days). Illness delay was shorter when patients recognised they were having a stroke. Six patients called for help themselves; in 27 cases a family member called for help; and in seven cases it was a member of the public. In 32 cases the general practitioner was called; an ambulance was called in seven cases; and one patient went straight to the emergency department.

In the three non-stroke groups most people were aware of the symptoms of stroke and most $(72.5-90 \%)$ said they would call an ambulance. Overall, knowledge of stroke risk factors was better in these groups than in the stroke group. Recognition of major risk factors for stroke was poor in all groups. Ten of the 40 nurses did not recognise hypertension as a risk factor and 21 did not believe that smoking was a risk factor. Nine nurses said that stroke was only occasionally an emergency though most others (including 37 of the 40 members of the general public) thought it was always an emergency. In the at risk group there were six patients with atrial fibrillation and none of the six listed atrial fibrillation as a risk factor. Thirty had hypertension and only 13 of these gave hypertension as a risk factor. All four smokers recognised smoking as a risk factor.

In this population most people had some knowledge of stroke symptoms and considered it an emergency. Nevertheless fewer than half of the stroke patients were aware that they were having a stroke and an ambulance was called directly in only seven of the 40 cases. Patients experiencing a stroke may be reluctant to accept the fact and may delay calling for help. Most people's knowledge of stroke is derived from relatives, friends, and the media rather than from health services.

Both health professionals and the public need more education about the need for early treatment of acute stroke. The authors of this paper offer five points to be covered in education programmes. They are: the need for early admission to a stroke unit or hospital, symptom recognition by patients, the need to call for medical help quickly, the need to call an ambulance rather than a general practitioner, and the need for general practitioners called about acute stroke to expedite hospital admission.

A Journal of Neurology, Neurosurgery and Psychiatry 2004;75:567-571. 